\title{
ASYMPTOTIC EXPANSIONS FOR SOLUTIONS OF SMOOTH RECURRENCE EQUATIONS
}

\author{
SHING-WHU JHA, ATTILA MÁTÉ, AND PAUL NEVAI
}

(Communicated by J. Marshall Ash)

\begin{abstract}
Let $\left\langle y_{n}: n \geq 1\right\rangle$ be a convergent sequence of reals, where for each $n$ the tuple $\left\langle y_{n}, y_{n+1}, \ldots, y_{n}+k, 1 / n\right\rangle$ satisfies one of $r$ equations, depending on the residue class of $n(\bmod r)$, for some given $k$ and $r$. Assume these equations are smooth, they have the same gradient in the first $k+1$ variables, and this gradient satisfies a certain nonmodularity condition. We then show that $y_{n}$ has $r$ asymptotic expansions, depending on the residue class of $n(\bmod r)$, in terms of powers of $1 / n$. This result enables us to discuss the asymptotic behavior of the recurrence coefficients associated with certain orthogonal polynomials. A key ingredient in the proof of the main result is a lemma involving exponential sums.
\end{abstract}

\section{INTRODUCTION}

The aim of these notes is to prove the following:

Theorem 1. Let $k \geq 0$ and $m, r \geq 1$ be integers, and let $H_{l} \quad(0 \leq l<r)$ be complex-valued functions of $k+2$ real variables $x_{0}, \ldots, x_{k+1}$ all partial derivatives of order $\leq m$ of which are continuous in a neighborhood of the origin o. Assume that

$$
\partial_{j} H_{l}(\mathbf{o})=\partial_{j} H_{l^{\prime}}(\mathbf{o}) \quad\left(0 \leq j \leq k, 0 \leq l, l^{\prime}<r\right)
$$

$\left(\partial_{j}\right.$ abbreviates $\partial_{j} / \partial x_{j}$; we do not require that $\left.\partial_{k+1} H_{l}(\mathbf{o})=\partial_{k+1} H_{l^{\prime}}(\mathbf{0})\right)$. Writing $\lambda_{j}=\partial_{j} H_{0}(\mathbf{0})(0 \leq j \leq k)$, assume, further, that

$$
\sum_{j=0}^{k} z^{j} \lambda_{j} \neq 0
$$

Received by the editors April 10, 1989.

1980 Mathematics Subject Classification (1985 Revision). Primary 39A10, 41A60; Secondary 10G05, 42C05.

Key words and phrases. Asymptotic expansions, exponential sums, orthogonal polynomials, recurrence equations.

This material is based upon work supported by the National Science Foundation under Grant Nos. DMS-8801200 (second author) and DMS-8814488 (third author), by the PSC-CUNY Research Award Program of the City University of New York under Grant No. 668337 (second author), and by NATO (third author). 
holds for all complex numbers $z$ with $|z|=1$. Let the real numbers $y_{n}$ with

$$
\lim _{n \rightarrow \infty} y_{n}=0
$$

form a solution of the recurrence equations

$$
H_{l}\left(y_{n}, y_{n+1}, \ldots, y_{n+k}, 1 / n\right)=0
$$

for each $n \geq 1$, where $0 \leq l<r$ is determined by $n$ so that $l \equiv n(\bmod r)$. Then there are numbers $c_{l 1}, \ldots, c_{l m}(0 \leq l<r)$ such that

$$
y_{n}=\sum_{\nu=1}^{m} c_{l \nu} n^{-\nu}+o\left(n^{-m}\right) \quad(n \equiv l \quad(\bmod r))
$$

as $n \rightarrow \infty$. Moreover, the numbers $c_{l \nu}$ for $0 \leq l<r$ and $1 \leq \nu \leq m$ depend only on the $j$ th partial derivatives of $H_{l^{\prime}}$ for $1 \leq j \leq \nu$ and $0 \leq l^{\prime}<r$.

This result extends the Theorem of $[8, \mathrm{p} .423]$. There is a genuine need for this extension; the quoted result was used to obtain asymptotic expansions of recurrence coefficients associated with certain orthogonal polynomials. At the end of this paper we will give an example to which the quoted result is not applicable, while the above theorem is so. Another extension, to systems of recurrence equations, of the Theorem of [8] is found in [2, Theorem 1.1, pp. 209-210].

\section{Proof of the main Result}

A key lemma needed for the proof of Theorem 1 is the following.

Lemma 2. Let $\lambda_{j}(0 \leq j \leq k)$ be complex numbers such that

$$
\sum_{j=0}^{k} z^{j} \lambda_{j} \neq 0
$$

holds for all complex $z$ with $|z|=1$. Then the system of equations

$$
\sum_{l=0}^{r-1} x_{l} \sum_{\substack{j=0 \\ \nu+j \equiv l(\bmod r)}}^{k} \lambda_{j}=0 \quad(0 \leq \nu \leq r-1)
$$

is nonsingular, i.e. it has only the trivial solution.

The proof of this lemma makes use of exponential sums. In effect, we will factor the coefficient matrix of the above system of equations as the product of two nonsingular Vandermonde matrices.

Proof. Write

$$
e_{r}(x)=e^{2 \pi i x / r}
$$

this notation is common in number theory. Noting that

$$
\sum_{s=0}^{r-1} e_{r}(s q)= \begin{cases}r & \text { if } r \mid q, \\ 0 & \text { otherwise }\end{cases}
$$


we can write (7) as

$$
\sum_{l=0}^{r-1} x_{l} \sum_{j=0}^{k} \lambda_{j} \sum_{s=0}^{r-1} e_{r}(s(\nu+j-l))=0 \quad(0 \leq \nu \leq r-1) .
$$

Introducing the notation

$$
\Lambda_{s}=\sum_{j=0}^{k} \lambda_{j} e_{r}(s j) \quad(0 \leq s \leq r-1)
$$

and

$$
y_{s}=\Lambda_{s} \sum_{l=0}^{r-1} x_{l} e_{r}(-s l) \quad(0 \leq s \leq r-1),
$$

the system of equations in (8) can be written as

$$
\sum_{s=0}^{r-1} e_{r}(s \nu) y_{s}=0 \quad(0 \leq \nu \leq r-1) .
$$

Since the coefficients in this system of equations form a nonsingular Vandermonde matrix, it follows that $y_{s}=0$ for $0 \leq s \leq r-1$. Substituting this into (9) and noting that $\Lambda_{s} \neq 0$ for $0 \leq s \leq r-1$ in view of (6), it follows that

$$
\sum_{l=0}^{r-1} e_{r}(-s l) x_{l}=0 \quad(0 \leq s \leq r-1) .
$$

Again, the coefficient matrix is a nonsingular Vandermonde matrix, and so $x_{l}=$ 0 for $0 \leq l \leq r-1$. This is what we wanted to show. The proof is complete.

Next we turn to the proof of Theorem 1. Rather than giving all the details, we will emphasize the differences from the proof the Theorem in [8, pp. 425-427].

Proof of Theorem 1 . Notice that $H_{l}(\mathbf{0})=0$ by (3) and (4) for each $l$ with $0 \leq l<r$. Therefore, according to Taylor's formula,

$$
\begin{aligned}
H_{l}\left(y_{n}, y_{n+1}, \ldots, y_{n+k}, \frac{1}{n}\right)=\sum_{\nu=1}^{m-1} \frac{1}{\nu !}\left(\sum_{j=0}^{k} y_{n+j} \partial_{j}+\frac{1}{n} \partial_{k+1}\right)^{\nu} H_{l}(\mathbf{o}) \\
+\frac{1}{m !}\left(\sum_{j=0}^{k} y_{n+j} \partial_{j}+\frac{1}{n} \partial_{k+1}\right)^{m} H_{l}\left(\theta y_{n}, \theta y_{n+1}, \ldots, \theta y_{n+k}, \frac{\theta}{n}\right) \\
\quad(0 \leq l<r, n \equiv l(\bmod r))
\end{aligned}
$$

holds for some $\theta$ with $0<\theta<1$, provided $n$ is large enough (so that the point $\left(y_{n}, y_{n+1}, \ldots, y_{n+k}, 1 / n\right)$ belongs to a convex neighborhood of $\mathbf{0}$ in which $H_{l}$ is $m$ times continuously differentiable). The left-hand side here is zero according to (4). In view of the continuity of the $m$ th derivatives of $H_{l}$ at $\mathbf{0}$, (3) implies that the right-hand side will change only slightly if we replace the argument of $H_{l}$ with $\mathbf{o}$ in the last term; estimating the magnitude 
of this change, we obtain the following (note that the modified last term of the preceding formula being incorporated into the sum below, $\nu$ now goes to $m$ rather than $m-1)$ :

$$
\sum_{\nu=1}^{m} \frac{1}{\nu !}\left(\sum_{j=0}^{k} y_{n+j} \partial_{j}+\frac{1}{n} \partial_{k+1}\right)^{\nu} H_{l}(\mathbf{o})=o\left(\sum_{j=0}^{k}\left|y_{n+j}\right|^{m}+n^{-m}\right),
$$

as $n \rightarrow \infty(n \equiv l(\bmod r))$.

Using induction on $m$, we may assume that

$$
y_{n}=\sum_{\nu=1}^{m-1} c_{l \nu} n^{-\nu}+\delta_{n} \quad(0 \leq l<r, n \geq 1, \text { and } n \equiv l \quad(\bmod r)),
$$

where

$$
\delta_{n}=o\left(n^{-m+1}\right)
$$

as $n \rightarrow \infty$. Indeed, for $m=1,(12)$ is justified by (3), and for $m>1$ it is justified by the induction hypothesis saying that (5) is valid with $m-1$ replacing $m$. Substituting (11) and (12) into (10), we obtain in exactly the same way as formula (17) of $[8$, p. 426] was obtained that

$$
\begin{aligned}
C_{l m}^{\prime} n^{-m}+\sum_{j=0}^{k} \delta_{n+j} \lambda_{j}=o\left(\sum_{j=0}^{k}\left|\delta_{n+j}\right|\right) & +o\left(n^{-m}\right) \\
& (n \rightarrow \infty, n \equiv l(\bmod r)) .
\end{aligned}
$$

In fact, the only difference between this formula and formula (17) of [8] is the dependence of $C_{l m}^{\prime}$ on $l$.

Choose $c_{l m}(0 \leq l<r)$ as the solution of the system of equations

$$
\sum_{l=0}^{r-1} c_{l m} \sum_{\substack{j=0 \\ \nu+j \equiv l(\bmod r)}}^{k} \lambda_{j}=-C_{\nu m}^{\prime} \quad(0 \leq \nu<r) .
$$

This system of equations is solvable according to Lemma 2. Put

$$
f(n)=\delta_{n}-c_{l m} n^{-m} \quad(0 \leq l<r, n \geq 1, \text { and } n \equiv l \quad(\bmod r)) .
$$

In order to complete the proof of Theorem 1, we only have to show that

$$
f(n)=o\left(n^{-m}\right) \text {. }
$$

Indeed, if we show this, then (5) becomes valid in view of (11) and (15).

To show (16), observe that according to (13)-(15) we have

$$
\sum_{j=0}^{k}\left(f(n+j)+o\left(n^{-m}-(n+j)^{-m}\right)\right) \lambda_{j}=o\left(\sum_{j=0}^{k}\left|\delta_{n+j}\right|\right)+o\left(n^{-m}\right)
$$

as $n \rightarrow \infty$. Using the Lemma of [8, p. 424], this equation implies (16); the details are similar to the verification of $(20)$ in $[8$, p. 427]. 


\section{AN APPLICATION TO ORTHOGONAL POLYNOMIALS}

Let $\gamma_{n}(n \geq 0)$ be the leading coefficient of the $n$th orthonormal polynomial on the real line with respect to the weight function $|x|^{\rho} \exp \left(-x^{6} / 6\right)(\rho>-1)$ and write $a_{n}=\gamma_{n-1} / \gamma_{n}\left(a_{n}=0\right.$ for $\left.n \leq 0\right)$. Then the recurrence equation

$$
\begin{aligned}
a_{n}^{2}\left(a_{n-2}^{2}+a_{n-1}^{4}\right. & +2 a_{n-1}^{2} a_{n}^{2}+a_{n}^{4}+a_{n-1}^{2} a_{n+1}^{2} \\
+ & \left.2 a_{n}^{2} a_{n+1}^{2}+a_{n+1}^{4}+a_{n+1}^{2} a_{n+2}^{2}\right)=n+\rho \frac{1+(-1)^{n}}{2}
\end{aligned}
$$

is satisfied for all $n \geq 0$ (see [4, Lemma 1, p. 93]). Freud showed that

$$
\lim _{n \rightarrow \infty} a_{n}(10 / n)^{1 / 6}=1
$$

(see $[5$, p. 6]), and conjectured much more; his conjectures have now been settled (see [6] and [7]). We obtained an asymptotic expansion for $a_{n}$ in terms of powers of $1 / n$ in [8, pp. 427-428] in case $\rho=0$; in fact, later, with W. C. Bauldry and T. Zaslavsky we obtained asymptotic expansions for the recurrence coefficients of a much larger class of orthogonal polynomials (see e.g. [2, Theorem 5.1, p. 223] and [11, Theorem 1, p. 496]). However, we have not been able to handle the case $\rho \neq 0$ until now. Theorem 1 with $r=2$ enables us to fill this gap.

Writing $F\left(a_{n+j}:-2 \leq j \leq 2\right)$ for the left-hand side of (17), and putting $y_{n}=a_{n}(10 / n)^{1 / 6}$ and

$$
H_{l}\left(x_{j}:-2 \leq j \leq 3\right)=F\left(x_{j}\left(1+j x_{3}\right)^{1 / 6}:-2 \leq j \leq 2\right)-1-\rho x_{3} \frac{1+(-1)^{l}}{2}
$$

for $l=0,1,(17)$ becomes

$$
\begin{aligned}
H_{l}\left(y_{n-2}, y_{n-1}, y_{n}, y_{n+1}, y_{n+2},\right. & 1 / n)=0 \\
& (l=0,1, n \equiv l(\bmod 2)),
\end{aligned}
$$

which is analogous to (4), but the point $\mathbf{o}$ has to be replaced with $\mathbf{p}=\langle 1,1,1$, $1,0\rangle$, in view of (18). Using Theorem 1 , we obtain for all integers $m \geq 0$ that

$$
a_{n}\left(\frac{10}{n}\right)^{1 / 6}=\sum_{\nu=0}^{m} c_{l \nu} n^{-\nu}+o\left(n^{-m}\right) \quad(l=0,1, n \equiv l \quad(\bmod 2))
$$

as $n \rightarrow \infty$, where $c_{0}, c_{1}, \ldots$ are appropriate constants. The details of the arguments used to derive (21) are similar to those in [8, pp. 427-428].

Asymptotic series of the form (21) have many uses for orthogonal polynomials, e.g. when studying the distribution of zeros of orthogonal polynomials or the differential equations satisfied by some orthogonal polynomials. The enhanced usefulness of Theorem 1, as shown in the above example, in comparison to that of the Theorem of $[8$, p. 423] extends several of the applications given in the literature of the latter result. For example, in view of Theorem 1, the Theorem of $[9$, p. 746] and its generalization, the Theorem of [10, p. 302], now apply to the orthogonal polynomials on the real line associated with the 
Freud-type measure $|x|^{\rho} \exp \left(-x^{m}\right) d x$ for every even integer $m>0$ and every real $\rho>-1$, whereas until now this was known only in the case $\rho=0$. (Cf. the Conjecture in [9, p. 750]. Theorem 1 settles this Conjecture for $m$ a positive even integer, but it is still open for other positive real values of $m$; the reason for this is that the recurrence coefficients of these polynomials are not known to satisfy a recurrence formula of the type given in (17) if $m$ is not an even integer.) Results of the papers [3], [12], and [13] appear to generalize in a similar fashion. There is a difficulty in extending the results of [1] in this way. Namely, this paper generalizes the results of [3] to asymmetric Freud weights, but for these weights a recurrence formula of the type (17) is not known in the case $\rho \neq 0$.

\section{REFERENCES}

1. W. C. Bauldry, Estimates for asymmetric Freud polynomials, J. Approx. Theory 63 (1990) (to appear).

2. W. C. Bauldry, A. Máté, and P. Nevai, Asymptotics for solutions of systems of smooth recurrence equations, Pacific J. Math. 133 (1988), 209-227.

3. S. S. Bonan and D. S. Clark, Estimates of the Hermite and Freud polynomials, J. Approx. Theory 63 (1990) (to appear).

4. G. Freud, On the greatest zero of an orthogonal polynomial, Acta Sci. Math. 24 (1973), 91-97.

5. _ On the coefficients in the recursion formulae of orthogonal polynomials, Proc. Roy. Irish Acad. Sec. A 76 (1976), 1-6.

6. D. S. Lubinsky, H. N. Mhaskar, and E. B. Saff, Freud's conjecture for exponential weights, Bull. Amer. Math. Soc. 15 (1986), 217-221.

7. _ A proof of Freud's conjecture for exponential weights, Constructive Approximation 4 (1988), 65-83.

8. A. Máté and P. Nevai, Asymptotics for the greatest zeros of orthogonal polynomials, Proc. Amer. Math. Soc. 93 (1985), 423-429.

9. A. Máté, P. Nevai, and V. Totik, Asymptotics for the zeros of orthogonal polynomials, SIAM J. Math. Anal 17 (1986), 745-751.

10. __ Asymptotics for the zeros of orthogonal polynomials associated with infinite intervals, J. London Math. Soc. 33 (1986), 303-310.

11. A. Máté, P. Nevai, and T. Zaslavsky, Asymptotic expansions of ratios of orthogonal polynomials with exponential weights, Trans. Amer. Math. Soc. 287 (1985), 495-505.

12. P. Nevai, Orthogonal polynomials associated with $\exp \left(-x^{4}\right)$, Second Edmonton Conference on Approximation Theory, Conference Proceedings 3, Canadian Mathematical Society, Ottawa, 1983, pp. 263-265.

13. _ Asymptotics for orthogonal polynomials associated with $\exp \left(-x^{4}\right)$, SIAM J. Math. Anal. 15 (1984), 1177-1187.

(S.-W. Jha, P. Nevai) Department of Mathematics, The Ohio State University, ColumBUS, OHIO 43210-1174

(A. Máté) Department of Mathematics, Brooklyn College of the City University of NEW YORK, BROOKLYN, NeW YORK 11210 